\title{
. Understanding How in Situ Generated Hydrogen Controls the 2 Morphology of Platinum Nanoparticles
}

\author{
${ }_{3}$ Neus Aguilera-Porta, ${ }^{\dagger, \ddagger, \S, \|}$ Monica Calatayud, ${ }^{*}, \dagger, \downarrow, \perp$ Caroline Salzemann, ${ }^{\S, \|}$ and Christophe Petit $*, \S, \|$ \\ $4{ }^{\dagger}$ Sorbonne Universités, UPMC Univ Paris 06, UMR 7616, Laboratoire de Chimie Théorique, F-75005 Paris, France \\ 5 "CNRS, UMR 7616, Laboratoire de Chimie Théorique, F-75005 Paris, France \\ $6{ }^{8}$ Sorbonne Universités, UPMC Univ Paris 06, UMR 8233, Laboratoire MONARIS, F-75005 Paris, France \\ 7 "CNRS, UMR 8233, Laboratoire MONARIS, F-75005 Paris, France \\ ${ }_{8}^{\perp}$ Institut Universitaire de France, F-75252 Paris, France
}

9 S Supporting Information

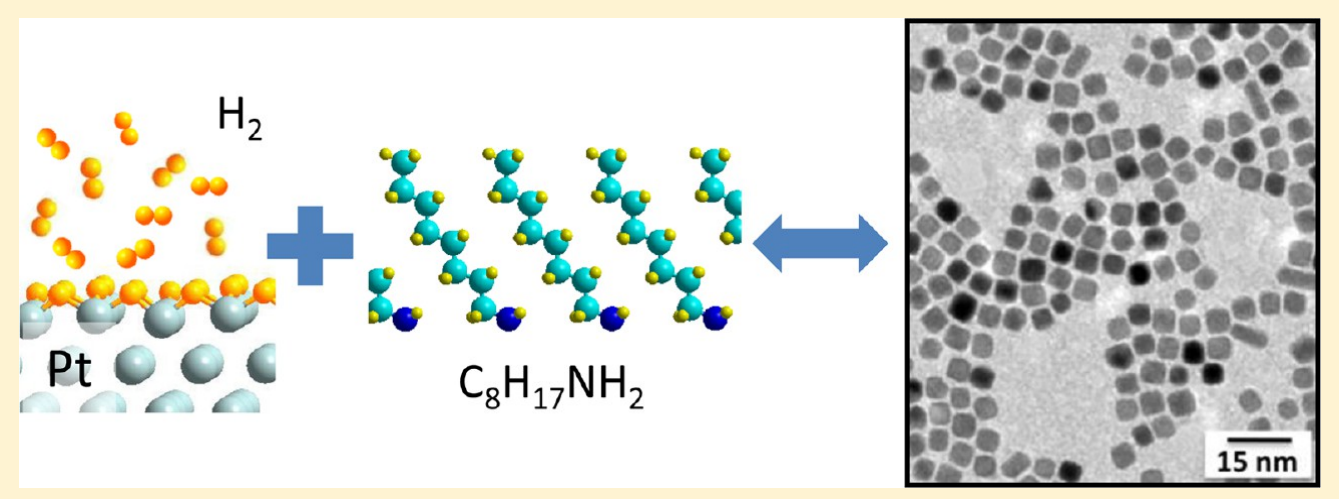

10 ABSTRACT: Small adsorbed molecules play a key role in the morphology of inorganic nanoparticles. The presence of in situ 11 generated hydrogen during the synthesis of platinum nanoparticles is found to drive the growth of cubic nanocrystals, but little is 12 known about the processes occurring at the molecular level. In this paper, we use standard ab initio calculations to show that 13 hydrogen preferentially adsorbs on (100) Pt facets compared to (111) stabilizing the cubic morphology. Moreover, we provide 14 experimental and theoretical evidence that moderate partial pressure of hydrogen is needed to obtain nanocubes. In the absence 15 of hydrogen, or for low partial pressures, small nanoparticles with undefined shape are formed; however, longer exposure to 16 hydrogen pressure around 1 atm leads to the formation of cubes. Finally, this theoretical result allows presenting an experimental 17 protocol to be used to obtain platinum nanocubes with different degree of truncation.

\section{$18 \square$ INTRODUCTION}

19 Many physical and chemical properties depend on the shape of 20 the particles forming the material, ${ }^{1}$ hence the challenge of 21 controlling the crystal morphology in a wide range of scientific 22 and technological applications. The chemical route is largely used 23 to control the shape of inorganic nanocrystals, although there is 24 still no theoretical drawback of the main forces driving it. Despite 25 many studies found in the literature, the theoretical and 26 computer simulation of nanoparticles' synthesis and growth are 27 still in the initial stage of development, and most of these works 28 do not take into account the complexity of the chemical 29 synthesis. It is still paramount to explain the mechanisms at the 30 origin of the uniformity of shape. Indeed, the field is still wide 31 open to future research aiming at identifying the conditions to 32 control the nanomorphology.

33 In the chemical synthesis of the nanocrystals, the chemical 34 bath possesses a complex composition containing various 35 reactants, solvents, surfactants, (counter)ions, and impurities. 36 Moreover, during the reaction, byproducts can be formed, which could play a role in the nuclei formation and the following 37 nanoparticles' growth. ${ }^{2}$ For instance, the role of dissolved gas $\mathrm{H}_{2} 38$ on the shape control of platinum nanocrystals has recently been 39 demonstrated experimentally. ${ }^{3}$ Some authors also report the role 40 of halide, ligands, or adsorbed CO on the nanomorphology. ${ }^{4-6}{ }_{41}$ Besides, the role of the initial shape of the nanocrystals and the 42 growth kinetics have been emphasized. ${ }^{7,8}$ Beyond the exper- 43 imental recipe, a theoretical approach is needed to rationalize and 44 predict the stability of the nano-objects. In the present paper, the 45 goal is to emphasize the role of a byproduct generated during the 46 chemical synthesis in the final nanoparticle morphology. We 47 focus here on the specific case of platinum. We use ab initio 48 calculations to explain how the presence of in situ generated $\mathrm{H}_{2} 49$ drives the formation of cubic nanoparticles. We also explain the 50 impact of the experimental conditions, namely, the partial 51

Received: March 3, 2014

Revised: $\quad$ April 7, 2014 
52 pressure of hydrogen and the order of addition of reactants in the 53 chemical bath: the nanocubes are obtained only following a 54 particular experimental protocol (i) when the surfactant agent is 55 added after the reducing hydrogen and (ii) in the presence of 56 moderate hydrogen partial pressure. If one of these conditions is 57 not satisfied, nanowires made of aggregated nanospheres are 58 obtained. Interestingly, it is possible to obtain nanocubes with a 59 selected degree of truncation by controlling the time of 60 extraction of the nanoparticles. Beyond the specific case of the 61 platinum, we believe that this approach based on theory and 62 experiment allows gaining further understanding of the shape 63 control of metallic nanocrystals obtained by the chemical route, 64 and furthermore, this approach may be a powerful tool of general 65 application in the field of nanocrystal synthesis.

66 The morphology of a given crystal is governed by the stability 67 of the interface between the solid phase and the media. The 68 crystal grows following the most stable surface termination. 69 Therefore, the interaction of the substrate-adsorbate system is 70 crucial to understand mechanisms of stabilization for a given 71 morphology. A way of predicting the morphology of a crystal is 72 thus to calculate the energies of the different surfaces, the 73 predominant surface will be that of lower energy. Different 74 approaches based on the Wulff construction exist to predict the 75 crystalline shape (for instance, see ref). Pt crystallizes in the face76 centered cubic system which has two major surface terminations: 77 (111) and (100). Predominance of the former will lead to 78 octahedral particles, whereas the latter leads to cubic shapes. In 79 this paper, we present an approach to calculate the surface energy 80 of (111) and (100) terminations in different conditions (bare, 81 hydrogenated, and capped by a surfactant molecule) by standard 82 periodic density functional theory (DFT) calculations. The 83 factors stabilizing each termination will be analyzed, and the 84 results will be compared with the particles synthesized under 85 different conditions. A protocol to obtain cubic particles with a 86 degree of truncation will be proposed.

\section{EXPERIMENTAL SECTION}

88 Pt nanocrystals are synthesized by the phase transfer synthesis 89 method presented by Brust et al. ${ }^{10}$ Typically, it consists of metal 90 ion transfer from an aqueous phase to an organic phase using an 91 extractant molecule. The nanocrystals are obtained by chemical 92 reduction. This method separates the nucleation media (the 93 water/toluene interface) from the growth media (toluene) 94 yielding to a well-dispersed NP organic solvent. ${ }^{11}$

95 The metallic salt solution is prepared as follows: aqueous 96 solutions of $3.3 \times 10^{-2} \mathrm{M} \mathrm{PtCl}_{4}$ were prepared by dissolving the 97 metallic salt in acid media $\left(1 / 3 \mathrm{HCl} 37 \%\right.$ to $\left.2 / 3 \mathrm{H}_{2} \mathrm{O}\right)$ to form 98 the complexes $\mathrm{H}_{2} \mathrm{PtCl}_{6}$. After stirring during $1 \mathrm{~h}$, this aqueous 99 solution is then mixed with $1.9 \times 10^{-2} \mathrm{M}$ tridecylammonium 100 bromide (TDAB) dispersed in $80 \mathrm{~mL}$ of toluene. To ensure 101 maximum transfer of metal ions from the water phase to the 102 organic phase, the transferring agent $(\mathrm{TDAB})$ was present in 103 large excess (corresponding to $\mathrm{TDAB} / \mathrm{PtCl}_{4}=3 / 1$ ) and the 104 extraction occurs in four steps; TDAB/toluene solution $(20 \mathrm{~mL})$ 105 is added to the acidic solution containing the metallic salt, the 106 two-phases mixture was vigorously stirred until all the metallic 107 complexes were transferred into the organic phase $(30 \mathrm{~min})$. The 108 organic phase is collected and $20 \mathrm{~mL}$ of TDAB/toluene is added 109 again. Finally, the four fractions are combined. The colorless 110 water phase is then discarded.

111 Chemical Reduction of the Metallic Salt. Procedure I: 112 Reduction in the Presence of the Capping Agent. Initially, $1136.10^{-3} \mathrm{~mol}$ of octylamine $\left(\mathrm{C}_{8} \mathrm{H}_{17} \mathrm{NH}_{2}\right)$ capping agent $(\mathrm{CA})$ is added under stirring to $10 \mathrm{~mL}$ of the obtained organic solution 114 containing the metallic complexes. A freshly prepared aqueous 115 solution of $\mathrm{NaBH}_{4}\left(10 \mathrm{~mL}, 1 \mathrm{~mol} \cdot \mathrm{L}^{-1}\right)$ is dropwise added under 116 stirring, yielding to an emulsion. This induces the reduction of 117 the metallic salt at the interface of the organic and aqueous 118 phases. Both the reducing agent and the capping agent are added 119 in large stoichiometric excess relative to the platinum $\left(\mathrm{NaBH}_{4} / 120\right.$ $\mathrm{Pt}^{4+}=160: 1$ and $\left.\mathrm{CA} / \mathrm{Pt}=96: 1\right)$. The emulsion turns relatively 121 fast from orange to dark brown. The reaction takes place for one 122 night after what the stirring has removed, and the organic phase, 123 containing the metal nanoparticles, is collected and evaporated 124 using a Rotavapor. The black paste obtained is then washed with 125 $40 \mathrm{~mL}$ of ethanol. Then, the turbid solution is centrifuged, and 126 the supernatant is discarded. This operation is repeated twice. 127 After the last centrifugation, the precipitate is redispersed in $4 \mathrm{~mL} \quad 128$ of toluene. The final solution contains the coated $\mathrm{C}_{8} \mathrm{NH}_{2}{ }_{129}$ nanoparticles in toluene.

130

Procedure II: Reduction Followed by the Addition of 131 Capping Agent. In this case, the freshly prepared aqueous 132 solution of $\mathrm{NaBH}_{4}\left(10 \mathrm{~mL}, 1 \mathrm{~mol} \cdot \mathrm{L}^{-1}\right)$ is dropwise added under 133 stirring to $10 \mathrm{~mL}$ of the organic solution containing the metallic 134 complexes. The reduction occurs in the emulsion phase, at the 135 interface of the organic and aqueous phases, which results in a 136 color change from orange to dark brown. After $60 \mathrm{~min}$ (ripening 137 time $\left.\tau_{\text {rip }}\right), 6.10^{-3}$ mol of octylamine is then added. The chemical 138 bath is kept under stirring for one night, and then the 139 nanocrystals are extracted following the procedure described 140 above. The final solution contains the coated $\mathrm{C}_{8} \mathrm{NH}_{2}$ nano- 141 particles in toluene.

142

Environmental Effect. The synthesis of Pt NCs has been 143 performed both in the presence and in the absence of hydrogen 144 atmosphere under glovebox with outgassed solutions. 145

Hydrogen is naturally produced by the chemical reduction. To 146 perform the reaction under saturated $\mathrm{H}_{2}$ atmosphere, a closed 147 screw cap with three ports is used, and both the reducing and the 148 capping agent are injected through a silicone septum. In order to 149 see the influence of hydrogen atmosphere on the nanoparticles, 150 we performed the same syntheses with open screw cap and by 151 bubbling $\mathrm{N}_{2}$ into the chemical bath, during and after the 152 reduction, to drive out the hydrogen formed.

In order to see the influence of the overpressure of $\mathrm{H}_{2}$ (see 154 Discussion and Figure 7), the capping agent is added at a specific 155 time, not using the silicon septum but opening the vessels. This 156 yields to a drastic decrease of the overpressure. 157

The TEM micrographs are obtained on a JEOL 1011158 apparatus, HRTEM are obtained on a JEOL 2010.

Computational Methods and Models. The VASP 160 code $^{12,13}$ is used with the revised Perdew-Becke-Erzernhof 161 functional rPBE. The core electrons are represented by 162 pseudopotentials generated by the projector augmented wave 163 (PAW) method. ${ }^{14,15}$ The valence electrons (Pt: 10; N: 5; C: 4; 164 H: 1) are described by plane-wave basis sets with a cutoff of 400165 $\mathrm{eV}$. A $5 \times 5 \times 1 \mathrm{k}$-points scheme is used in the Brillouin zone. The 166 following unit cells were used $(100): c 2 \times 2,5.635 \times 5.635 \times 35167$ $\AA^{3}$; $(111): 2 \times 2,5.628 \times 5.628 \times 35 \AA^{3}$, including a vacuum of 168 $\sim 20 \AA$ to prevent interaction between successive slabs. The 169 thickness of the slabs is five layers, each layer containing four $\mathrm{Pt} 170$ atoms, see Figure 2. The unit cells are chosen to have similar 171 surface area and equal composition of the slab (20 atoms each) so 172 that the total energy can be easily compared. The three 173 uppermost Pt layers are relaxed during the optimization 174 procedures together with the adsorbates, and the two bottom 175 layers are frozen to the ideal bulk positions. The ionic 176 

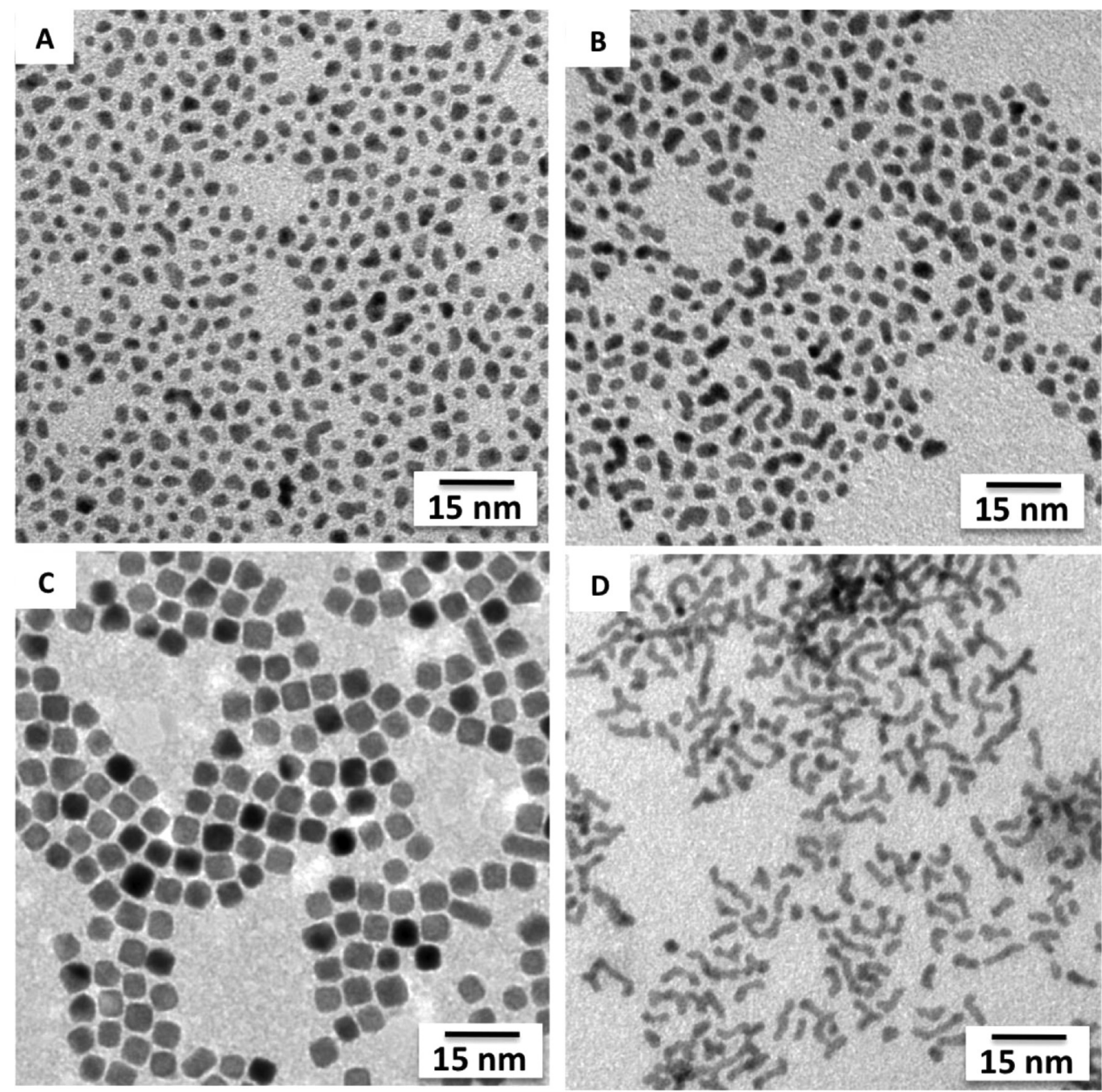

Figure 1. TEM images of platinum nanocrystals coated by octylamine and redispersed in toluene. A drop of solution is deposited on a TEM grid. (A) and (B) Synthesis made following the procedure I (i.e., capping agent added before the reducing agent) in the presence (A) or in the absence (B) of hydrogen. (C) and (D) Synthesis made following the procedure II (i.e., capping agent added after the reducing agent) in the presence (C) or in the absence (D) of hydrogen.

177 convergence is achieved for total energy until the difference was 178 below $1 \mathrm{meV}$; the conjugate gradient method was employed. 179 Periodic DFT has been successfully used in the past to describe 180 adsorption systems and gold self-assembled monolayers. ${ }^{16,17}$ 181 Dispersion forces have been included as implemented in the 182 Grimme-D2 approach, ${ }^{18}$ for the adsorbates and the first slab layer 183 as in ref 19. The parameters used are given as Supporting 184 Information.

185 We consider the reaction of adsorption between the slab and $\mathrm{N}$ 186 molecules of type M (hydrogen or octylamine)

$187 \quad \mathrm{slab}+\mathrm{N} \cdot \mathrm{M}=[\mathrm{slab}-\mathrm{NM}]$

188 The stability of a given slab may be calculated as the reaction 189 energy associated with eq 1

$190 \Delta G_{\mathrm{r}}=\mathrm{G}_{[\mathrm{slab}-\mathrm{NM}]}-\mathrm{G}_{\mathrm{slab}}-\mathrm{N} \mu_{\mathrm{M}}$

191 where $G_{\mathrm{i}}$ are the Gibbs free energies of the covered and bare 192 slabs, $\mu_{\mathrm{M}}$ is the chemical potential of the adsorbate M. Because we 193 are interested in the relative stability between bare and covered 194 slabs, volume and entropy changes can be neglected assuming 195 that they are equal for the two slabs. The Gibbs energy can then 196 be replaced by the internal energy of each slab as directly 197 obtained from the total-energy calculations. We have chosen the 198 (111) termination as reference for the bare slab, and the $1 / 2 \mathrm{H}_{2}$ (or octylamine) gas-phase energy as reference for the covered 199 slabs.

\section{RESULTS AND DISCUSSION}

201

Platinum nanocrystals (NCs) are obtained from chemical 202 reduction by an aqueous solution of sodium borohydrate 203 $\left(\mathrm{NaBH}_{4}\right.$, reducing agent) of a metallic salt solubilized in 204 toluene. ${ }^{3,20}$ To stabilize the nanoparticles, octylamine molecules 205 $\left(\mathrm{C}_{8} \mathrm{H}_{17} \mathrm{NH}_{2}\right.$, surfactant agent denoted as $\left.\mathrm{C}_{8} \mathrm{NH}_{2}\right)$ are used as 206 passivating agent that can be added either before (procedure I) 207 or $1 \mathrm{~h}$ after (procedure II) the addition of the reducing agent. ${ }^{3}$ In 208 such a synthesis, the chemical reduction takes place at the water/ 209 oil interface under stirring which allows separating the nucleation 210 and the growth steps, yielding to calibrated nanocrystals with low 211 size dispersion. ${ }^{11}$ In this synthesis, the passivating agent is weakly 212 bound to the metallic surface (physisorbed) and the reaction 213 produces hydrogen spontaneously. Thus, a competition occurs 214 between $\mathrm{C}_{8} \mathrm{NH}_{2}$ and $\mathrm{H}_{2}$ in interaction with the metallic surface, 215 allowing the size and shape control of the nanoparticles. ${ }^{3}$ In order 216 to investigate such competition, the order of addition considered 217 in procedures I and II has been studied, whereas the role of the in 218 situ generated hydrogen has been evidenced by carrying out 219 synthesis in the presence and in the absence of hydrogen. The 220 presence of a moderated pressure of $\mathrm{H}_{2}$ is achieved by working 221 with closed vessels, whereas the absence of $\mathrm{H}_{2}$ is studied by 222 
(100) Top view

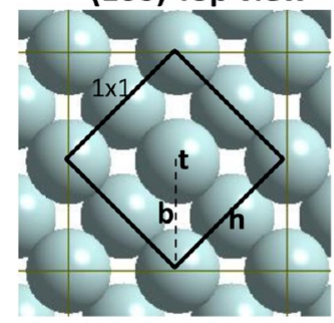

(111) Top view

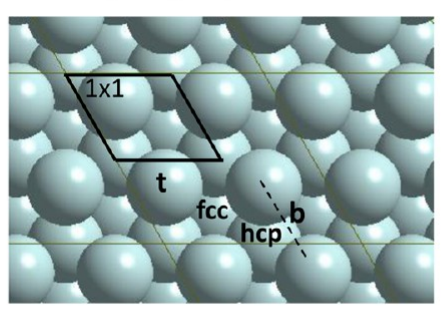

(100) Side view

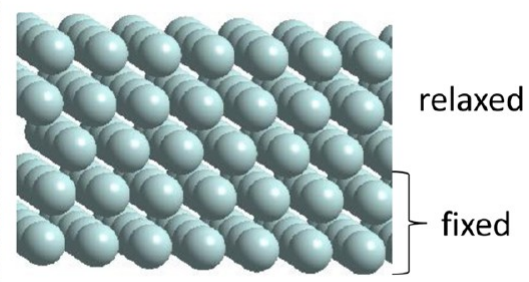

(100)
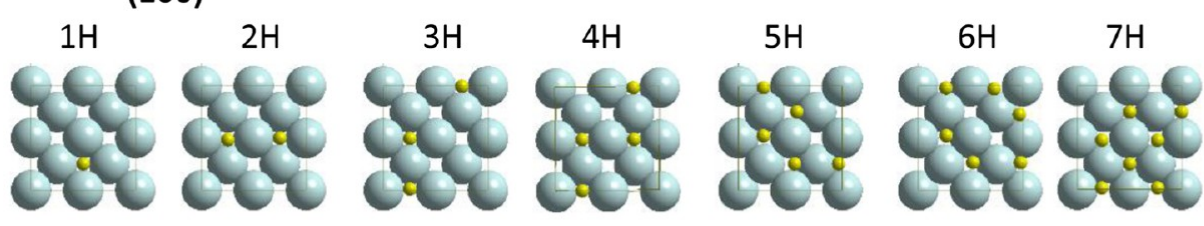

$8 \mathrm{H}$

(111)
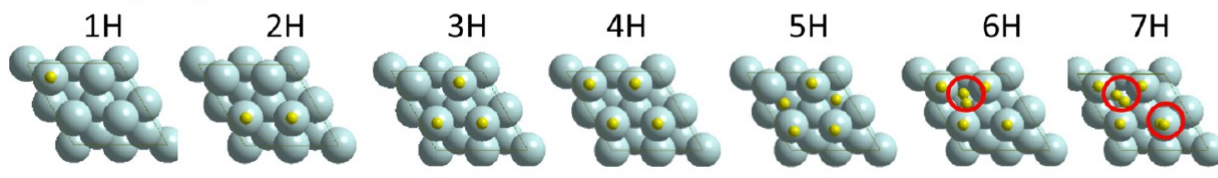

$8 \mathrm{H}$

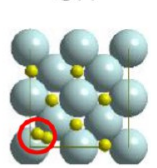

Figure 2. Most stable structures for bare slabs (top) and hydrogen-covered slabs (bottom). The $1 \times 1$ unit cells are displayed: $b$, bridging; $t$, top; h, hollow. Red circles indicate $\mathrm{H}_{2}$ molecules.

223 bubbling $\mathrm{N}_{2}$ during the reaction that drives out the hydrogen 224 formed.

225 Figure 1 shows the coated $\mathrm{C}_{8} \mathrm{NH}_{2}-\mathrm{Pt} \mathrm{NCs}$ obtained after 226 extraction and redispersion in toluene. To minimize the external 227 factors, they are synthesized under glovebox with outgassed 228 solutions, as $\mathrm{O}_{2}$ perturbs the formation of platinum nanocryst$229 \mathrm{als}^{20}$ The Pt nanoparticles obtained from procedure $\mathrm{I}$ in the 230 presence (Figure 1A) or in the absence (Figure 1B) of hydrogen 231 are characterized by a mean diameter of around $2.4 \mathrm{~nm}$ and a size 232 dispersion of $21 \%$. No clear size and shape effect is observed 233 between both samples. In contrast, the synthesis performed by 234 procedure II leads to drastic changes of both size and shape of the 235 nanoparticles. Indeed, $4.5 \mathrm{~nm}$ Pt nanocubes having 13\% size 236 dispersion are obtained in the presence of hydrogen (Figure 1C), 237 and platinum nanowires characterized by a cross sectional 238 diameter of $2.0 \mathrm{~nm}$ have been obtained without hydrogen 239 (Figure 1D). Thus, the synthesis conditions (i.e., the presence or 240 absence of $\mathrm{H}_{2}$ in the solution) together with the order of addition 241 of the capping agent, strongly influence the platinum nano242 morphology. In the following, we will investigate theoretically 243 the respective role of these different elements on the shape 244 control.

245 Periodic DFT calculations have been carried out to compute 246 the stability of the terminations of (111) and (100) slabs. The 247 bare (111)-terminated slabs are found to be more stable than the 248 (100)-terminated ones in agreement with the literature, ${ }^{21}$ and 249 thus in vacuum conditions, octahedral particles are expected to 250 be formed. For the covered slabs, we have considered the 251 interaction of hydrogen and of the surfactant octylamine. For the 252 hydrogen case, we consider the adsorption of 1 to 8 hydrogen 253 atoms on the slabs. Experiments and calculations find that there 254 exist low barrier pathways for the dissociation of dihydrogen on $255 \mathrm{Pt}^{22-24}$ so we consider only atomic hydrogen. Figure 2 displays 256 the optimized systems for all the compositions considered. It is shown that the (100) slab shows a high affinity for adsorbing 257 atomic $\mathrm{H}$ : $\mathrm{Up}$ to $7 \mathrm{H}$, the slab is covered by $\mathrm{H}$. For higher 258 coverage, the adsorbed atoms recombine to form $\mathrm{H}_{2}$ that leaves 259 the surface, whereas the hydrogenated (111) termination is 260 stable only up to $5 \mathrm{H}$. Beyond this value, $\mathrm{H}_{2}$ is formed. The higher 261 affinity of hydrogen for (100) with respect to (111) is also 262 observed in the calculated adsorption energies shown in Table 1, 263 t1 Figure S1, where the adsorption per $\mathrm{H}$ atom is always more 264 exothermic for (100) than for (111). The calculated adsorption 265 energy values are in agreement with those reported in the 266

Table 1. Calculated Adsorption Energy for the Hydrogenated and Octylamine Covered Slabs ${ }^{a}$

$\begin{array}{lcccl} & E_{\text {ads }}(111) & E_{\text {ads }}(100) & \begin{array}{c}E_{\text {ads }}(100) / \\ (111)\end{array} & \text { predicted shape } \\ 0 \mathrm{H} & -112.35^{c} & -110.49^{c} & 0.98 & \begin{array}{l}\text { truncated } \\ \text { octahedron } \\ \end{array} \\ & & & & \text { cube } \\ 1 \mathrm{H} & -0.41 & -0.53 & 1.29 & \text { cube } \\ 2 \mathrm{H} & -0.38 & -0.52 & 1.36 & \text { cube } \\ 3 \mathrm{H} & -0.29 & -0.52 & 1.77 & \text { cube } \\ 4 \mathrm{H} & -0.35 & -0.51 & 1.47 & \text { cube } \\ 5 \mathrm{H} & -0.21 & -0.46 & 2.17 & \text { cube } \\ 6 \mathrm{H} & -0.23^{b} & -0.41 & 1.76 & \text { cube } \\ 7 \mathrm{H} & -0.17^{b} & -0.37 & 2.20 & \text { cube } \\ 8 \mathrm{H} & -0.16^{b} & -0.31^{b} & 1.95 & \text { truncated } \\ \mathrm{C}_{8} \mathrm{NH}_{2} & -1.16 & -1.19 & 1.03 & \text { octahedron }\end{array}$

${ }^{a}$ Values are in $\mathrm{eV}$, calculated as $E_{\text {ads }}=\left(E_{\mathrm{NM}}-E_{\text {slab }}-\mathrm{NE}_{\mathrm{M}}\right) / \mathrm{N}$, where $\mathrm{M}$ is $1 / 2 \mathrm{H}_{2}$ or $\mathrm{C}_{8} \mathrm{NH}_{2}$ molecule calculated in the gas phase, and $E_{\text {slab }}$ refers to the reference energy for each slab. $E_{\text {ads }}(100) /(111)$ measures the relative stability of the two terminations: values $>1$ indicate predominance of cubic, values $<1$ indicate predominance of octahedral particles, and values close to 1 indicate truncated cubes. ${ }^{b}$ Molecular $\mathrm{H}_{2}$ is formed. ${ }^{c}$ Total energy. 
(100)
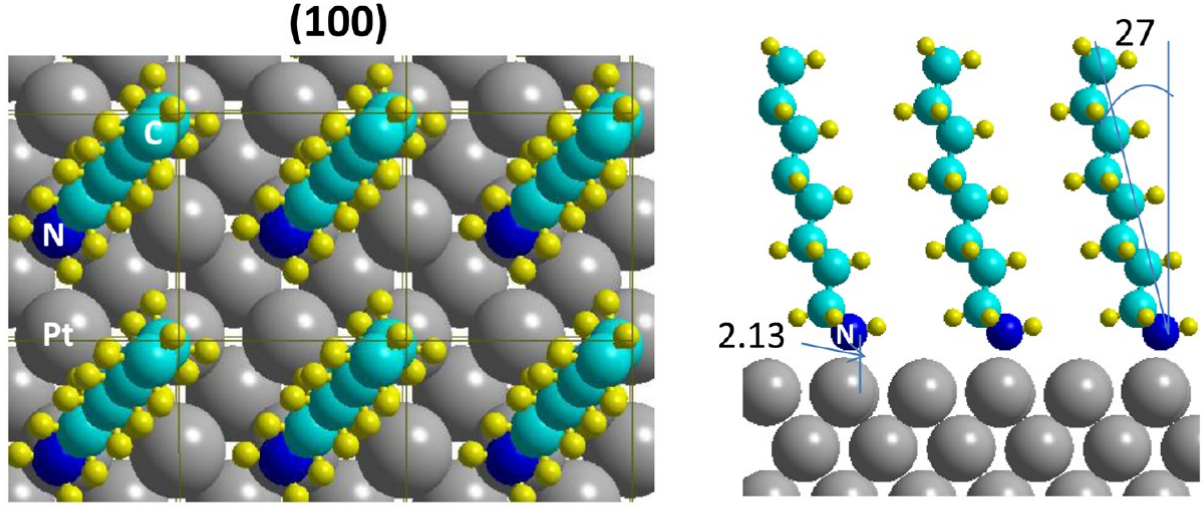

(111)
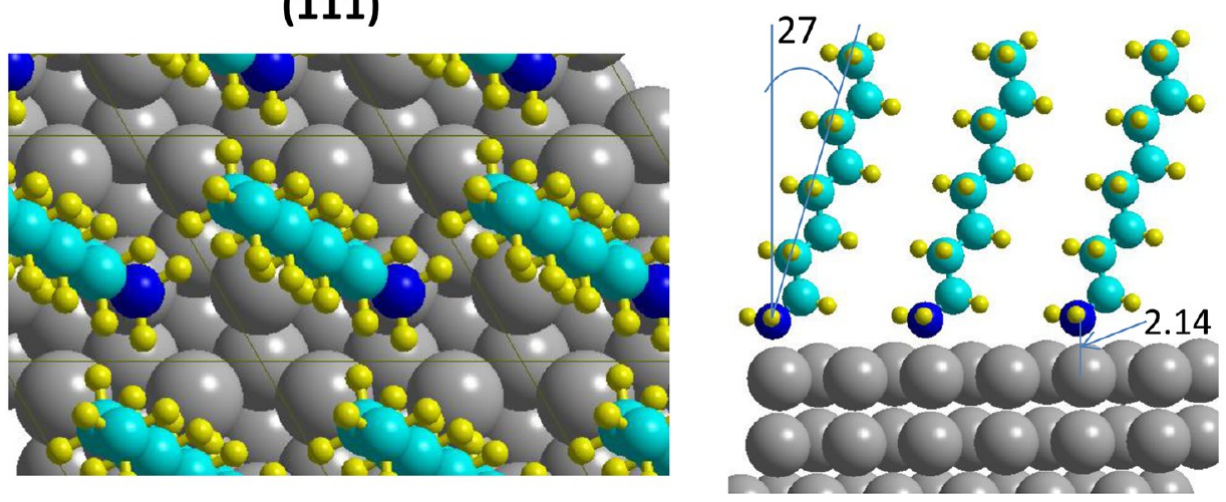

Figure 3. Top and side views of the $\mathrm{C}_{8} \mathrm{NH}_{2}$ molecule adsorbed on the model slabs. Angles in degrees, $\mathrm{N}-\mathrm{Pt}$ distance in $\AA$.

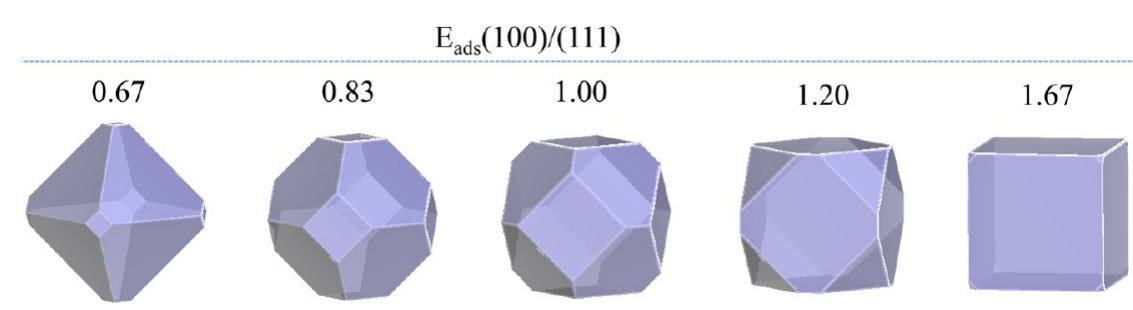

Figure 4. Morphology of $\mathrm{Pt}$ particles predicted from ab initio calculations. The adsorption energy of a reactant on each slab serves to compute the exposed area of each termination. The ratio between the adsorption energies $E_{\text {ads }}(100) /(111)$ is a measure of the shape: the particle is cubic (values higher than 1.6), octahedral (values lower than 0.7 ), or truncated (intermediate values).

267 literature for similar computational approaches. ${ }^{25,26}$ As regards 268 the adsorption site, hydrogen is found to adsorb on bridging sites 269 for the (100) slab and a mixture between bridge, top, and hollow 270 sites for the (111) slab, in agreement with previous 271 calculations. $^{25}$

272 The adsorption of octylamine is also computed for 273 comparison. The molecule is found to adsorb on quasi-top 274 sites in both surfaces, tilted $27^{\circ}$ from the vertical of the slab (see 275 Figure 3 ). The calculated adsorption energies for the two slabs 276 shown in Table 1 are very similar: $-1.16 \mathrm{eV}(111)$ and $-1.19 \mathrm{eV}$ 277 (100). These results indicate that $\mathrm{C}_{8} \mathrm{NH}_{2}$ adsorbs more strongly 278 than hydrogen on the $\mathrm{Pt}$ surfaces. Contrary to the hydrogen 279 adsorption, the octylamine molecule has no clear preference for a 280 given termination. In the following, we will focus on the 281 hydrogen adsorption, because experiments and theoretical 282 results highlight its crucial role in the synthesis.

283 The shape of platinum nanoparticles can be qualitatively 284 predicted from the $a b$ initio calculated adsorption energies. The 285 most exposed planes will be those growing more slowly (i.e., the 286 most stable). Taking the ratio of the adsorption energies on each of the slabs, $E_{\mathrm{ads}}(100) /(111)$ can be used as a measure of the 287 relative stability of the (100) slab with respect to (111). A sketch 288 of the so-calculated shapes is displayed in Figure 4. For $289 \mathrm{f} 4$ $E_{\text {ads }}(100) /(111)$ values close to 1 , the two terminations are 290 almost equivalent in energy and will expose the same area leading 291 to truncated octahedral morphology. As the $E_{\text {ads }}(100) /(111) 292$ value becomes higher than 1 , the (100) termination becomes 293 more stabilized, and the particle tends to form a cubic shape; the 294 opposite behavior leads to octahedral-shaped particles. 295

In order to compare the relative stability of the slabs under the 296 same external conditions we have carried out a thermodynamic 297 analysis. The stability of a given slab in the presence of an external 298 reactant $M$ may be calculated as the reaction energy associated 299 with eq la

$$
\begin{aligned}
& \text { slab }+\mathrm{NM}=[\mathrm{slab}-\mathrm{NM}] \\
& \Delta G_{\mathrm{r}}=G_{[\text {slab-NM }]}-G_{\text {slab }}-\mathrm{N} \mu_{\mathrm{M}}
\end{aligned}
$$

where $G_{\mathrm{i}}$ are the Gibbs free energies of the covered and bare 303 slabs, $\mu_{\mathrm{M}}$ is the chemical potential of the reactant M, N being its 304 

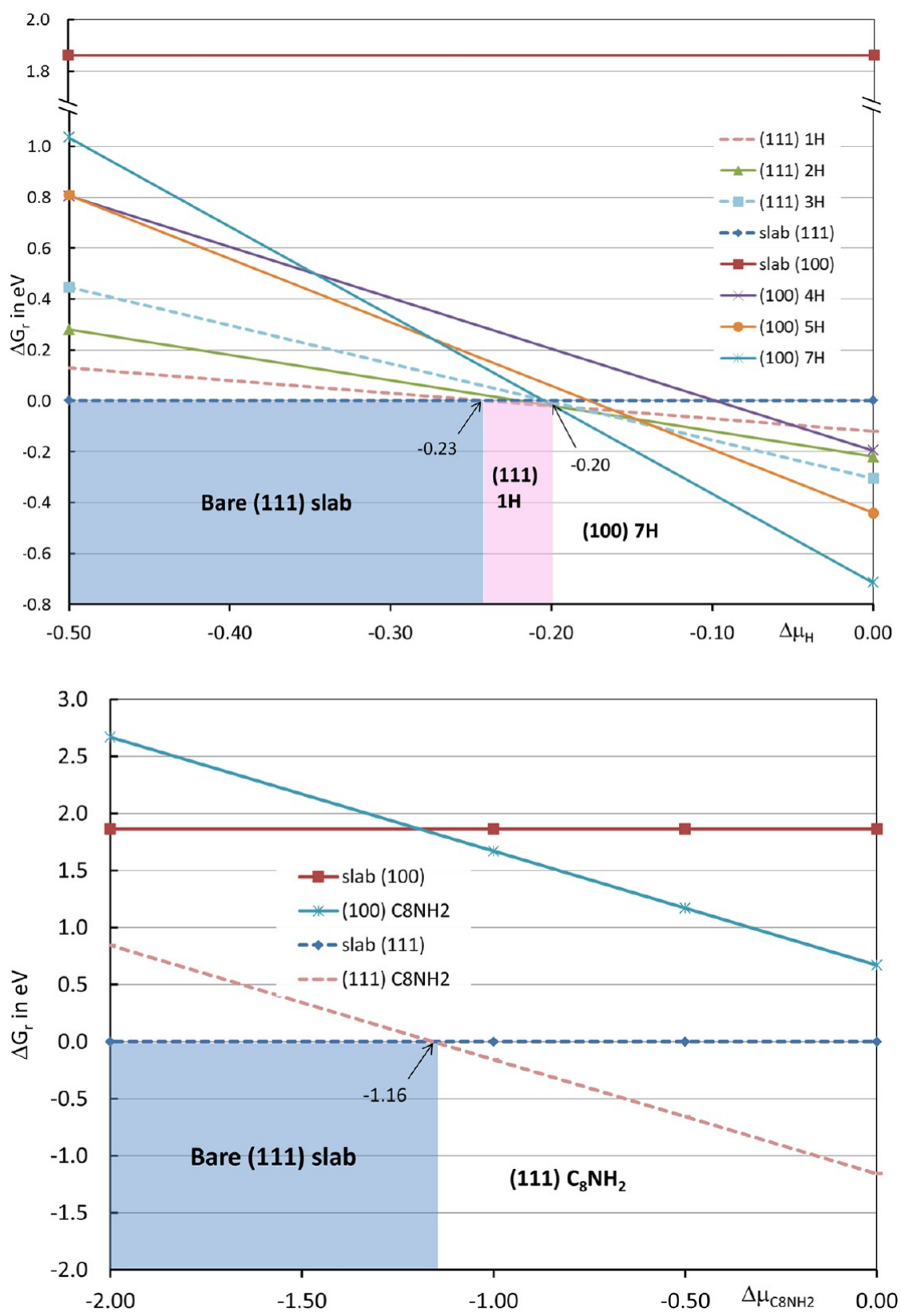

Figure 5. Diagrams of $\Delta G$ vs $\Delta \mu$ for the $\mathrm{H}$-covered and $\mathrm{C}_{8} \mathrm{NH}_{2}$ covered slabs. The most stable system corresponds to the lowest energy line. For the $\mathrm{H}$ covered slabs, the chemical potential represented here is relative to $1 / 2 \mathrm{H}_{2}$ and is denoted as $\Delta \mu_{\mathrm{H}}$, indicating that only $\Delta \mu_{\mathrm{H}}<0$ are meaningful (above this value $\mathrm{H}_{2}$ would condensate).

305 stoichiometric coefficient. This formulation allows a direct 306 comparison of the most stable surfaces for different composition: 307 a straight line with slope $-\mathrm{N}$ can be plotted as a function of $\mu_{\mathrm{M}}$ for 308 different content in M. For a given chemical potential, the 309 predominant surface will be that of lower energy. This approach 310 is well-suited to our case study, because in the experimental 311 synthesis, the chemical environment is the same for the two 312 terminations (111) and (100). The $\Delta G$ diagrams for all the 313 hydrogen-covered calculated slabs are presented in Figure S2. 314 We have represented the most stable hydrogenated slabs for the 315 two terminations taking as reference the most stable (111) slab in 316 Figure 5. It can be observed that for low (very negative) 317 hydrogen chemical potentials, the most stable system is the bare 318 (111) slab. At $\Delta \mu_{\mathrm{H}}$ values between $-0.23 \mathrm{eV}$ and $-0.20 \mathrm{eV}$, the $3191 \mathrm{H}-(111)$ covered slab is the most stable, whereas for higher $\Delta \mu_{\mathrm{H}}$ 320 values, the $7 \mathrm{H}-(100)$ slab becomes the most stable system. These data allow us to make the following interpretations: (i) High 321 content of hydrogen is needed to hydrogenate the platinum 322 slabs. The $\Delta \mu_{\mathrm{H}}=-0.23 \mathrm{eV}$ is indeed a large value compared to 323 metal oxides $\left(-2.21 \mathrm{eV}\right.$ for $\mathrm{ZnO}$, for instance $\left.{ }^{27}\right)$. (ii) The most 324 stable hydrogenated slab corresponds to the (100) termination, 325 and the hydrogenated (111) slabs is stable only in a narrow range 326 of hydrogen chemical potentials (i.e., between -0.23 and -0.20327 $\mathrm{eV})$.

The chemical potential of $\mathrm{H}_{2}$ can be related to the external 329 pressure by means of eq 3

$$
\mu=\mu^{0}+K_{\mathrm{B}} T \ln \left(p / p^{0}\right)
$$

where $\mu$ is the chemical potential, $\mu^{0}$ is the standard chemical 332 potential (tabulated), $K_{\mathrm{B}}$ is the perfect gas constant, $T$ is the 333 temperature, and $\mathrm{p} / \mathrm{p}^{0}$ the pressure/standard pressure. Taking 334 


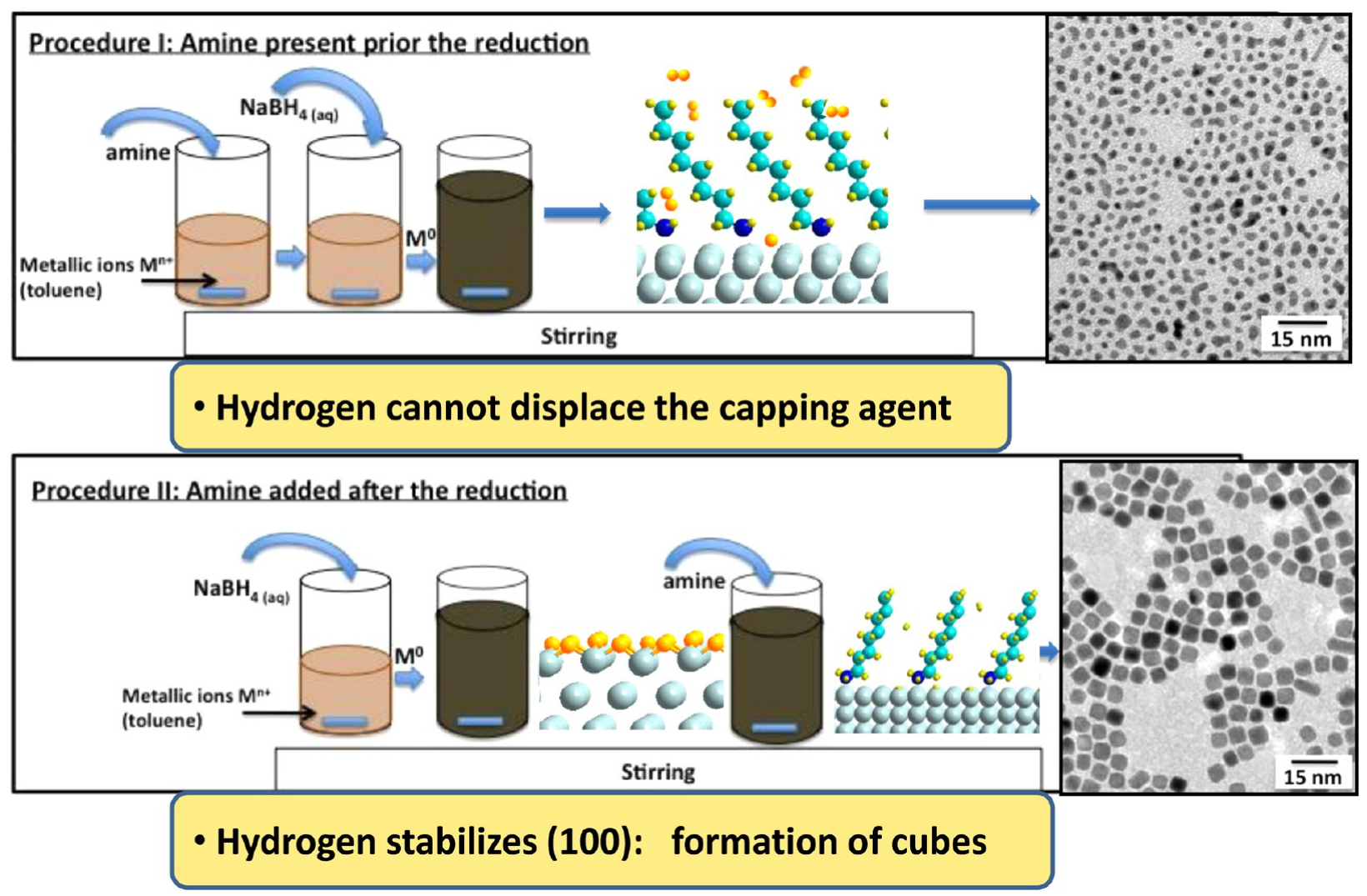

Figure 6. Scheme of the two experimental protocols used in the chemical synthesis. In procedure I, the capping agent is added first, so octylamine stabilizes the (111) termination; in a second step, the reducing agent is added, the hydrogen formed is not able to displace it, and small isotropic particles are formed. In procedure II, the addition of the reducing agent takes place first, and $\mathrm{H}_{2}$ is formed and preferentially stabilizes the (100) termination. The higher affinity of octylamine for the platinum surfaces displaces the hydrogen, and the cubic shape is preserved.

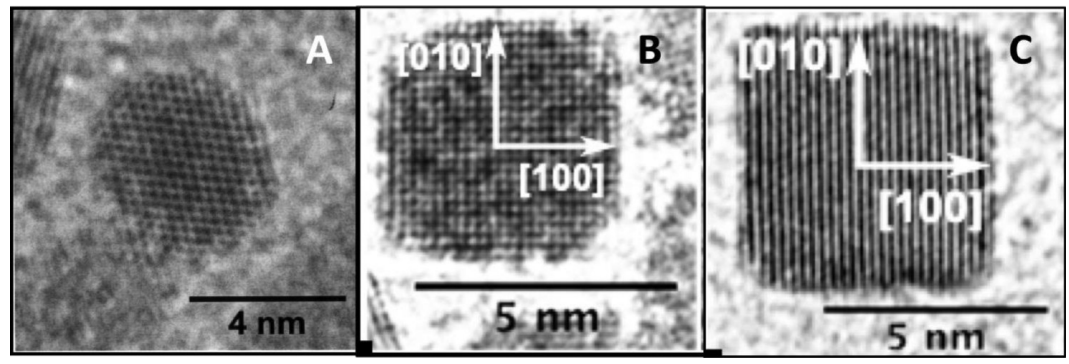

Figure 7. High-resolution TEM images of platinum nanocrystals coated by octylamine and redispersed in toluene. A drop of solution is deposited on a TEM grid. Syntheses are completed following the procedure II, but the capping agent is added (A) 45 min after the reducing agent, (B) 60 min after the reducing agent, and (C) 90 min after the reducing agent.

335 the value of chemical potential for which the (100) hydrogenated 336 slab starts being dominant $\Delta \mu_{\mathrm{H}}=-0.20 \mathrm{eV}$ (Figure 5), and at 337 room temperature, the corresponding pressure obtained by eq 3 338 is $1.18 \mathrm{~atm}$.

\section{DISCUSSION}

340 The results presented above allow us to explain two experimental 341 facts. First, the findings help explain the order of addition of the 342 reactants during the synthesis. In procedure I, the surfactant is 343 added before the reducing agent. The higher affinity of 344 octylamine for the platinum surfaces compared to hydrogen 345 (adsorption energy around $-1 \mathrm{eV}$ for $\mathrm{C}_{8} \mathrm{NH}_{2},-0.5 \mathrm{eV}$ for $\mathrm{H}_{2}$, 346 see Table 1) make the surfaces being covered by $\mathrm{C}_{8} \mathrm{NH}_{2}$. Because 347 octylamine has no preference for (111) or (100) surfaces, the 348 particles do not show a preferential termination. The particles 349 obtained by this procedure are therefore small and isotropic. In procedure II, the reducing agent is added first, and the in situ 350 generated hydrogen in contact with the metallic particles 351 stabilizes the (100) termination and promotes the formation of 352 cubic shapes. The subsequent addition of octylamine would 353 displace the surface hydrogen due to its higher affinity for 354 platinum, stabilizing the cubic shape. These results are 355 summarized schematically in Figure 6.

Second, moderate pressures of hydrogen are needed to obtain 357 the nanocubes, which means high chemical potential of 358 hydrogen. According to the $\Delta G$ diagram of Figure 5, at $\Delta \mu_{\mathrm{H}}=359$ $-0.20 \mathrm{eV}$, the (100) termination responsible for the cubic shape 360 starts being the most stable. Translating the chemical potential 361 into pressure using eq 3 gives a $\mathrm{H}_{2}$ pressure of $1.18 \mathrm{~atm}$. This 362 pressure is obtained experimentally by using closed vessels. 363 When the capping agent octylamine is added, it displaces 364 hydrogen on the surface and protects the particle. Hence, the 365 
366 nanocrystals grow in the [111] direction, yielding to the cubic 367 shape of the final product.

368 We can go further and explain the shape control from 369 truncated octahedron to cubic shape, depending on the moment 370 where the capping agent is added, as reported previously. ${ }^{20}$ 371 Figure 7 shows HR-TEM picture of nanocrystals obtained by 372 procedure II but waiting $45 \mathrm{~min}$ (Figure 7A), $60 \mathrm{~min}$ (Figure 373 7B), and 90 min (Figure 7C) before the addition of the capping 374 agent. In this procedure, the vessel is opened to introduce the 375 passivating agent, thus the hydrogen pressure strongly decreases, 376 and $\mathrm{H}_{2}$ is released. In fact, if we shorten the time at which the 377 passivating agent is introduced, the particles are no longer cubic 378 but have a much larger truncation. We interpret this result as 379 follows: the hydrogen pressure decreases upon the opening of 380 the vessel and so does the hydrogen chemical potential, inducing 381 a lower stabilization of the (100)-hydrogenated slab compared to 382 (111)-hydrogenated one. This fact promotes the truncation and 383 the formation of the cuboctahedron or truncated octahedron 384 shown in Figure 7. Therefore, the time of exposure to hydrogen 385 partial pressures is a way of controlling the degree of truncation 386 of the nanocubes.

\section{CONCLUSION}

388 The shape control in the chemical synthesis of platinum 389 nanoparticles has been studied by means of periodic DFT 390 calculations and experimental observations. The role of the in 391 situ generated hydrogen and of the surfactant used in the 392 synthesis is evidenced and explained with a theoretical surface 393 slab model. The adsorption energy calculated from DFT allows 394 understanding the preferential interaction of $\mathrm{H}$ with (100) 395 terminations, explaining why stable cubes can be obtained when $396 \mathrm{H}_{2}$ is present in the medium. Indeed, the external conditions of 397 moderate hydrogen pressure are needed and can be estimated 398 from the DFT calculation to be around $1 \mathrm{~atm}$. An experimental 399 protocol to obtain platinum nanocubes with a controlled degree 400 of truncation has been set up on the basis of the theoretical 401 results.

402 The above results are an illustration on how standard DFT 403 calculations can be used to rationalize the mechanisms stabilizing 404 nanoparticles on a molecular level. Theory can be used to 405 understand experimental results and may also help orienting the 406 experimentalists in the choice of reactants (selective interaction 407 with given terminations, strength of interaction with the slabs) 408 and experimental conditions (pressure, temperature, order of 409 addition of reactants). Such an approach opens the door to a 410 rational design of experiments beyond the trial-error procedure 411 often followed in chemical synthesis.

\section{ASSOCIATED CONTENT}

\section{S Supporting Information}

414 Evolution of the adsorption energy per hydrogen with the 415 hydrogen content (Figure S1), $\Delta G$ vs $\mu$ diagram for $\mathrm{H}$-covered 416 slabs (Figure S2), and dispersion D2 parameters used for the 417 calculations. This material is available free of charge via the 418 Internet at http://pubs.acs.org.

\section{AUTHOR INFORMATION}

\section{Corresponding Authors}

421 *E-mail: (M.C.) calatayud@lct.jussieu.fr. Tel.: +33144272505. 422 *E-mail: (C.P.) christophe.petit@upmc.fr. Tel.: +33 1442729 42306.

\section{Notes}

The authors declare no competing financial interest.

\section{ACKNOWLEDGMENTS}

426

SMART-IP2CT and Erasmus Program is acknowledged for 427 financial support (to N.A.-P.). This work was performed using 428 HPC resources from GENCI- CINES/IDRIS (grants 429 x2011082131, x2012082131, and x2013082131 for 2011- 430 2013, respectively) and the CCRE-DSI of Université P. M. 431 Curie. Boubakar Diawara is warmly acknowledged for providing 432 us with the Modelview visualization program.

\section{REFERENCES}

(1) Complex-Shaped Metal Nanoparticles: Bottom-up Syntheses and 435 ApplicationSau, T. K., Rogach, A. L. , Eds.; Wiley-VCH: Weinheim, 436 Germany, 2012.

(2) Petit, C.; Salzemann, C.; Demortière, A. Platinum and Paladium 438 Nanocrystals: Soft Chemistry Approach to Shape Control from 439 Individual Particles to their Self-assembled Superlattices. In Complex- 440 Shaped Metal Nanoparticles: Bottom-Up Syntheses and Application, Sau, 441 T. K., Rogach, A. L., Eds. Wiley-VCH: Weinheim, Germany, 2012. 442

(3) Salzemann, C.; Petit, C. Influence of Hydrogen on the Morphology 443 of Platinum and Palladium Nanocrystals. Langmuir 2012, 28, 4835- 444 4841.

(4) Chen, M.; Wu, B.; Yang, J.; Zheng, N. Small Adsorbate-Assisted 446 Shape Control of Pd and Pt Nanocrystals. Adv. Mater. 2012, 24, 862- 447 879.

(5) Bealing, C. R.; Baumgardner, W. J.; Choi, J. J.; Hanrath, T.; Hennig, 449 R. G. Predicting Nanocrystal Shape through Consideration of Surface- 450 Ligand Interactions. ACS Nano 2012, 6, 2118-2127.

(6) Chiu, C.-Y.; Li, Y.; Ruan, L.; Ye, X.; Murray, C. B.; Huang, Y. 452 Platinum Nanocrystals Selectively Shaped Using Facet-Specific Peptide 453 Sequences. Nat. Chem. 2011, 3, 393-399.

(7) Petroski, J. M.; Wang, Z. L.; Green, T. C.; El-Sayed, M. A. 455 Kinetically Controlled Growth and Shape Formation Mechanism of 456 Platinum Nanoparticles. J. Phys. Chem. B 1998, 102, 3316-3320. 457

(8) Xia, Y.; Xiong, Y.; Lim, B.; Skrabalak, S. E. Shape-Controlled 458 Synthesis of Metal Nanocrystals: Simple Chemistry Meets Complex 459 Physics? Angew. Chem., Int. Ed. 2009, 48, 60-103. 460

(9) Scopece, D. SOWOS: an Open-source Program for the Three- 461 Dimensional Wulff Construction. J. Appl. Crystallogr. 2003, 7, 21-26. 462

(10) Brust, M.; Walker, M.; Bethell, D.; Schiffrin, D. J.; Whyman, R. 463 Synthesis of Thiol-Derivatised Gold Nanoparticles in a Two-Phase 464 Liquid-Liquid System. J. Chem. Soc., Chem. Commun. 1994, 0, 801- 465 802.

(11) Petit, C.; Repain, V. Nucleation and Growth of Bimetallic 467 Nanoparticles. In Nanoalloys: Synthesis, Structure and Properties; 468 Alloyeau, D., Mottet, C., Ricolleau, C., Eds.; Springer Verlag: New 469 York, 2012; 1-24.

(12) Kresse, G.; Hafner, J. Ab Initio Molecular Dynamics for Liquid 471 Metals. Phys. Rev. B 1993, 47, 558.

(13) Kresse, G.; Hafner, J. Ab Initio Molecular-Dynamics Simulation of 473 the Liquid-Metal-Amorphous-Semiconductor Transition in Germa- 474 nium. Phys. Rev. B 1994, 49, 14251.

(14) Blochl, P. E. Projector Augmented-Wave Method. Phys. Rev. B 476 1994, 50, 17953-17979.

(15) Kresse, G.; Joubert, D. From Ultrasoft Pseudopotentials to the 478 Projector Augmented-Wave Method. Phys. Rev. B 1999, 59, 1758-1775. 479

(16) Luque, N. B.; Santos, E.; Andres, J.; Tielens, F. Effect of Coverage 480 and Defects on the Adsorption of Propanethiol on $\mathrm{Au}(111)$ Surface: A 481 Theoretical Study. Langmuir 2011, 27, 14514-14521.

(17) Tielens, F.; Costa, D.; Humblot, V.; Pradier, C. M. Character- 483 ization Of Omega-Functionalized Undecanethiol Mixed Self-Assembled 484 Monolayers on $\mathrm{Au}(111)$ : A Combined Polarization Modulation 485 Infrared Reflection-Absorption Spectroscopy/X-Ray Photoelectron 486 Spectroscopy/Periodic Density Functional Theory Study. J. Phys. 487 Chem. C 2008, 112, 182-190. 
489 (18) Grimme, S. Semiempirical GGA-type Density Functional 490 Constructed with a Long-Range Dispersion Correction. J. Comput. 491 Chem. 2006, 27, 1787.

492 (19) Mercurio, G.; McNellis, E. R.; Martin, I.; Hagen, S.; Leyssner, F.; 493 Soubatch, S.; Meyer, J.; Wolf, M.; Tegeder, P.; Tautz, F. S.; Reuter, K. 494 Structure and Energetics of Azobenzene on $\mathrm{Ag}(111)$ : Benchmarking 495 Semiempirical Dispersion Correction Approaches. Phys. Rev. Lett. 2010, $496104,036102$.

497 (20) Demortieres, A.; Launois, P.; Goubet, N.; Albouy, P. A.; Petit, C. 498 Shape-Controlled Platinum Nanocubes and Their Assembly into Two499 Dimensional and Three-Dimensional Superlattices. J. Phys. Chem. B 500 2008, 112, 14583-14592.

501 (21) Vitos, L.; Ruban, A. V.; Skriver, H. L.; Kollar, J. The Surface 502 Energy of Metals. Surf. Sci. 1998, 411, 186-202.

503 (22) Luntz, A. C.; Brown, J. K.; Williams, M. D. Molecular Beam 504 Studies of $\mathrm{H}_{2}$ and $\mathrm{D}_{2}$ Dissociative Chemisorption on $\mathrm{Pt}(111)$. J. Chem. 505 Phys. 1990, 93, 5240-5246.

506 (23) Olsen, R. A.; Kroes, G. J.; Baerends, E. J. Atomic and Molecular 507 Hydrogen Interacting with $\operatorname{Pt}(111)$. J. Chem. Phys. 1999, 111, 1115550811163.

509 (24) Pijper, E.; Kroes, G. J.; Olsen, R. A.; Baerends, E. J. The Effect of 510 Corrugation on the Quantum Dynamics of Dissociative and Diffractive 511 Scattering of $\mathrm{H}_{2}$ from $\mathrm{Pt}(111)$. J. Chem. Phys. 2000, 113, 8300-8312. 512 (25) Ford, D. C.; Xu, Y.; Mavrikakis, M. Atomic and Molecular 513 Adsorption on $\operatorname{Pt}(111)$. Surf. Sci. 2005, 587, 159-174.

514 (26) Ferrin, P.; Kandoi, S.; Nilekar, A. U.; Mavrikakis, M. Hydrogen 515 Adsorption, Absorption And Diffusion on and in Transition Metal 516 Surfaces: A DFT study. Surf. Sci. 2012, 606, 679-689.

517 (27) Meyer, B. First-Principles Study of the Polar O-terminated ZnO 518 Surface in Thermodynamic Equilibrium with Oxygen and Hydrogen. 519 Phys. Rev. B 2004, 69, 045416. 